\title{
Electron Beam Induced Domain Motion in Ferroelectric RKTP Observed By Transmission Electron Microscopy
}

\author{
James L. Hart ${ }^{1}$, Miryam Arredondo ${ }^{2}$, Mitra L. Taheri ${ }^{1}$ \\ 1. Department of Materials Science and Engineering, Drexel University, Philadelphia PA, US \\ 2. School of Mathematics and Physics, Queens University, Belfast Northern Ireland, UK
}

Utilization of ferroelectrics for practical applications requires precise control of domain structure, thus ferroelectric domain behavior is a widely researched area. In-situ transmission electron microscopy (TEM) is an increasingly popular tool for studying domain dynamics at high spatial and temporal resolution [1]-[3]. In this technique domain behavior is studied as a function of an applied electric field, however, the TEM's electron beam alone can induce ferroelectric domain motion independent of the applied field. Here we present electron beam induced domain motion in the uniaxial ferroelectric $\mathrm{Rb}$ doped $\mathrm{KTiOPO}_{4}$ (RKTP), using a JEOL $2100 \mathrm{LaB}_{6}$ TEM operated at $200 \mathrm{keV}$. This process is dependent on both the sample geometry and electron beam conditions. By manipulating the electron beam we can control the direction of domain propagation, and by using a condensed probe we can locally nucleate domains.

In TEM a negligible amount of beam electrons are trapped within the sample volume. Charging occurs due to Auger and secondary electron emission after inelastic scattering interactions [4]. For a conducting sample, charging is alleviated through compensating electric currents, but for an insulating material such as RKTP, large positive charge can accumulate beneath the electron beam leading to strong internal fields.

RKTP samples were prepared using a focused ion beam (FIB). All images shown here are DF images of 001 type reflections (Figure 1A). Initial domain morphology is shown in Figure 1B. Pre-existing domains are present, extending across the length of the sample with domain walls parallel to the c-axis (ferroelectric axis). When the sample is uniformly illuminated under the electron beam, the entire sample develops a positive charge. Because these samples were prepared in the FIB, there is a layer of conducting platinum on the top edge of the lift-outs. This metallic contact locally alleviates charging, leading to non-uniform charge build-up and a resultant internal electric field pointing towards the metal contact. This field acts to shrink pre-existing c- domains (Figures 2A and 2B). Conversely, if the specimen is non-uniformly illuminated so that only the top half of the sample is under the beam, only the top section positively charges, leading to an electric field pointing away from the top metal contact. Under such conditions we see nucleation and propagation of domains from the bottom of the sample up (Figure 2C). When the beam is condensed to a fine probe and placed in a mono-domain area (Figure $3 \mathrm{~A}$ ), only the illuminated region charges. The resultant electric field points radially away from the illuminated area. Because RKTP is a uniaxial ferroelectric, there is a single direction where the field is aligned with the ferroelectric axis allowing for domain nucleation. Under condensed beam conditions, we see domain nucleation at the perimeter of the electron beam (Figure 3B). This is the expected result as the beam perimeter has the highest charge density gradient and thus the strongest electric field [4].

This work is important for two reasons. Firstly, it shows that TEM observation of ferroelectrics can induce substantial domain motion. Such effects must be considered when analyzing results from in-situ 
TEM biasing experiments to ensure observed domain motion is a function of the applied field and not an artifact of beam induced fields. Secondly, this work demonstrates a possible mechanism for sub-micron scale ferroelectric control mediated through an electron beam. As shown here, a TEM beam can locally nucleate domains within the sample bulk or at an interface, and a TEM beam can dictate the direction of domain propagation based on beam location and sample geometry.

References:

[1] C. R. Winkler et al, Micron 43 (2012), p. 1121-2226

[2] C. R. Winkler et al, J. Appl. Phys 112 (2012)

[3] C. R. Winkler et al, Nano Letters 14 (2014), p. 3617-3622

[4] J. Cazaux, Ultramicroscopy 60 (1995), p. 411-425
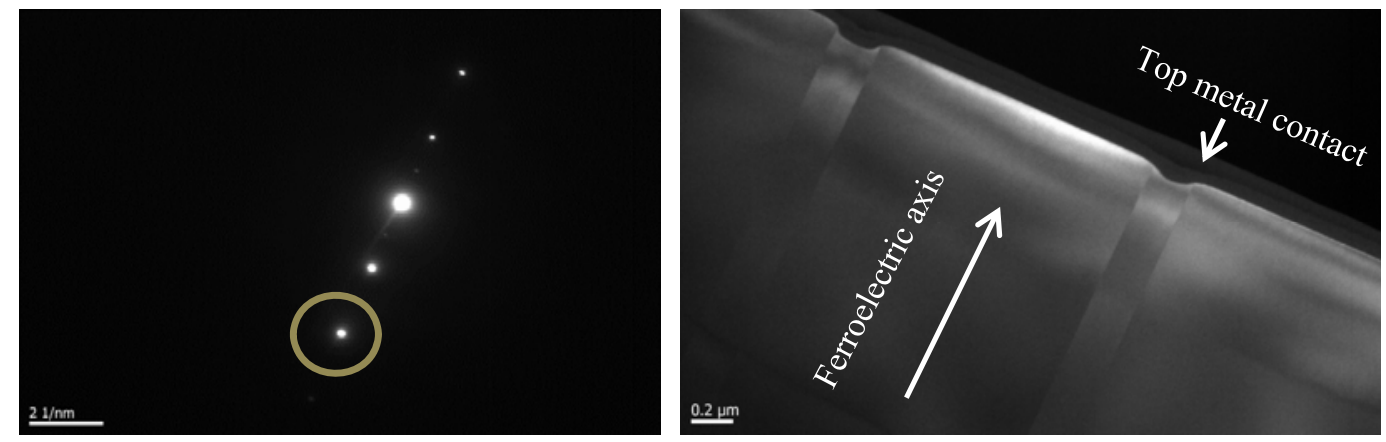

Figure 1. A. Two beam condition used to obtain DF images. B. Dark field TEM image of RKTP sample showing initial domain configuration.
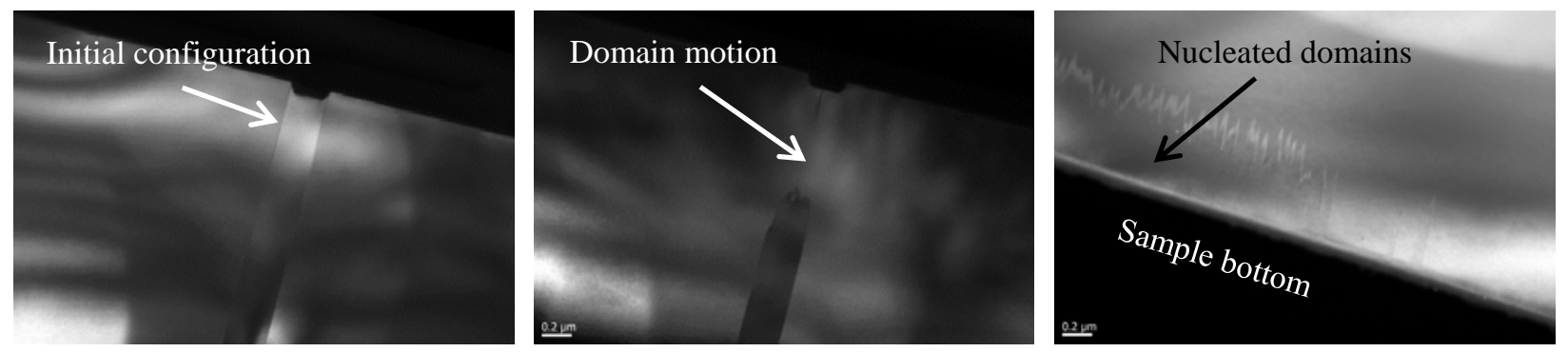

Figure 2. A. DF TEM image of initial RKTP domain. B. Downwards propagation of domain due to uniform sample illumination and charge dissipation through top metal contact. C. Nucleation and propagation of domains from the bottom of the sample up due to non-uniform illumination.
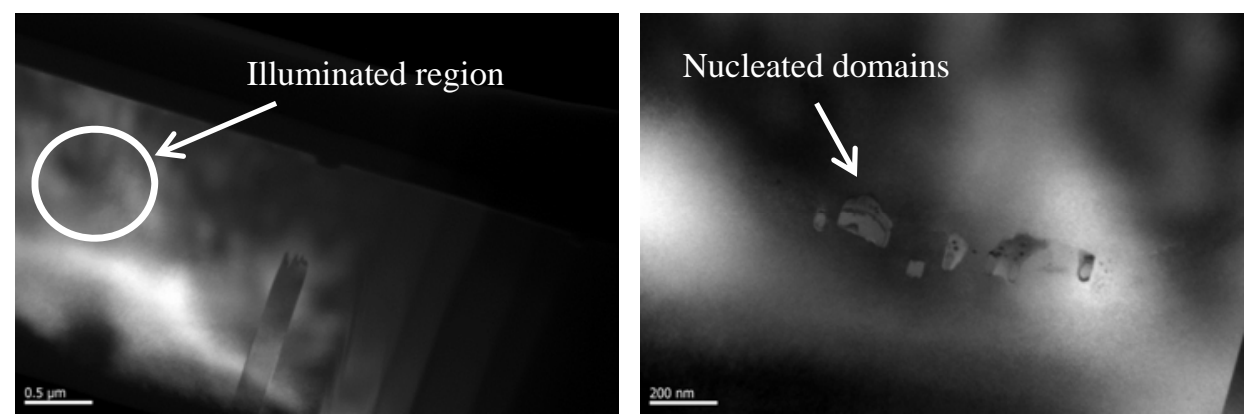

Figure 3. A. Image of monodomain area. Circle shows illuminated area. B. Domains which were locally nucleated due to the condensed beam. Nucleation occurred at perimeter of beam, shown in 3A. 